\title{
Candida Tropicalis Infection of the Knee Joint in an Immunocompromised Pediatric Patient: A Case Report
}

ISSN: 2578-0379

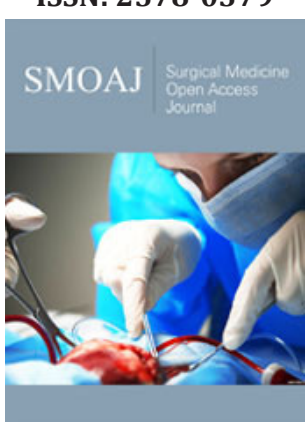

*Corresponding author: Benjamin Sookhoo, Department of Orthopedic Surgery, Jack Hughston Memorial Hospital, Medical College of Georgia at Augusta University, USA

Submission: 眥 March 2, 2020

Published: 僵March 17, 2020

Volume 3 - Issue 4

How to cite this article: Christopher D Muncie, Benjamin Sookhoo, Mudassar Khan. Candida Tropicalis Infection of the Knee Joint in an Immunocompromised Pediatric Patient: A Case Report. Surg Med Open Acc J.3(4). SMOAJ.000566.2020. DOI: 10.31031/SMOAJ.2020.03.000566.

Copyright@Benjamin Sookhoo, This article is distributed under the terms of the Creative Commons Attribution 4.0 International License, which permits unrestricted use and redistribution provided that the original author and source are credited.

\author{
Christopher D Muncie, Benjamin Sookhoo* and Mudassar Khan \\ Department of Orthopedic Surgery, Jack Hughston Memorial Hospital, USA \\ Department of Orthopedic Surgery, Medical College of Georgia at Augusta University, USA
}

\begin{abstract}
Background: Candida septic arthritis is a debilitating condition affecting joint function. Candida tropicalis, an organism found in normal human flora is noted to be the third most common pathogenic yeast in the elderly and immunosuppressed population. Infections are rare in the US, typically limited to the neonate and elderly populations. Most infections occur in the south America and southeast Asian regions. In the last 2 decades, Tropialis infection rates have risen impart due to antifungal resistance. We present a case of Candida tropicalis infection in the knee of a 13-year-old female with a past history of relapsed Acute Myeloid Leukemia following bone marrow transplant, pancytopenia and graft $v s$. host disease.
\end{abstract}

Methods: Patient case is thoroughly discussed, and a review of the current literature performed.

Result: Patient underwent multiple open irrigation and sharp debridement as well as arthroscopic complete synovectomy in addition to medical management. Patient remained pancytopenic throughout her course and eventually underwent granulocyte transfusion. During her course, she developed a relapse of her AML and subsequently expired. Limited case reports exist in the literature, with no recent reports of Candida arthritis in an adolescent in the United States.

Discussion: Candida septic arthritis remains a rare but potentially devastating condition, particularly in the immunocompromised patient population. Candida arthritis should be high on the treating physician's suspicion in immunocompromised patients presenting with new onset joint pain. Aggressive medical and surgical management is recommended, and current literature has shown a high cure rate with early treatment.

\section{Introduction}

Septic arthritis due to Candida species is a relatively rare infection typically seen in patients with immunocompromising conditions. While $C$. albicans is the most commonly encountered fungal species affecting humans, $C$. tropicalis has demonstrated increasing resistance to anti-fungal medications leading to persistent and difficult to control infections [1-15]. C. tropicalis is part of the normal human microbiota and is typically found on the skin and mucosal surfaces of the body. It is the third most common Non-Candida albicans Candida species (NCAC) isolated in clinical practice and primarily found in tropical regions, particularly Asia and South America [1,8]. Few reports of Tropicalis septic arthritis in North America have been published. We present a case report of a confirmed monoarticular $C$. tropicalis septic arthritis in a pediatric patient with chemotherapy induced granulocytopenia the setting of relapsed acute myeloid leukemia that lead to systemic candidiasis and patient mortality.

\section{Case Report}

Our patient is a 13-year-old Caucasian female who presented to our institution with relapsed AML following bone marrow transplant with clinical signs and symptoms concerning for graft vs. host disease. She had undergone previous chemotherapy treatment a little over a month prior to her admission and had an indwelling catheter. The patient was scheduled for intrathecal chemotherapy administration and was undergoing preoperative evaluation when it was noticed she had a diffuse rash and endorsing febrile episodes as well as diffuse joint pain. Pre-operative labs showed of white count of 0.1 cells $/ \mathrm{mm}^{3}$ as well as elevated inflammatory markers. She was admitted to our Children's hospital for further work up and evaluation. 2 
days into her hospital stay, patient awoke in the middle of the night new onset severe left knee pain and swelling with inability to bear weight to the extremity. An MRI of the knee (Figure 1), showed a moderate effusion to the joint. An arthrocentesis was performed and synovial fluid culture demonstrated fungal growth that was identified as C. tropicalis.

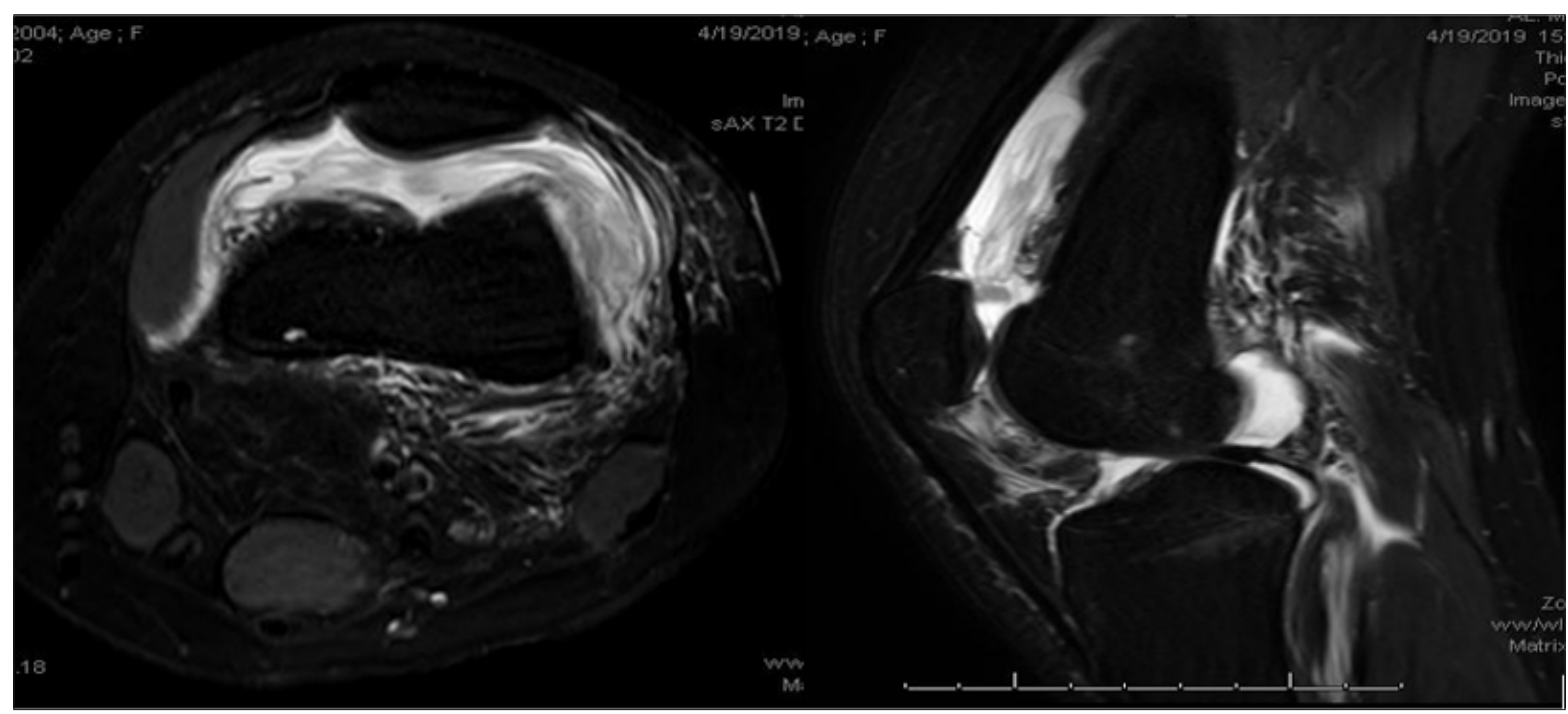

Figure 1: MRI of the knee showing moderate effusion.
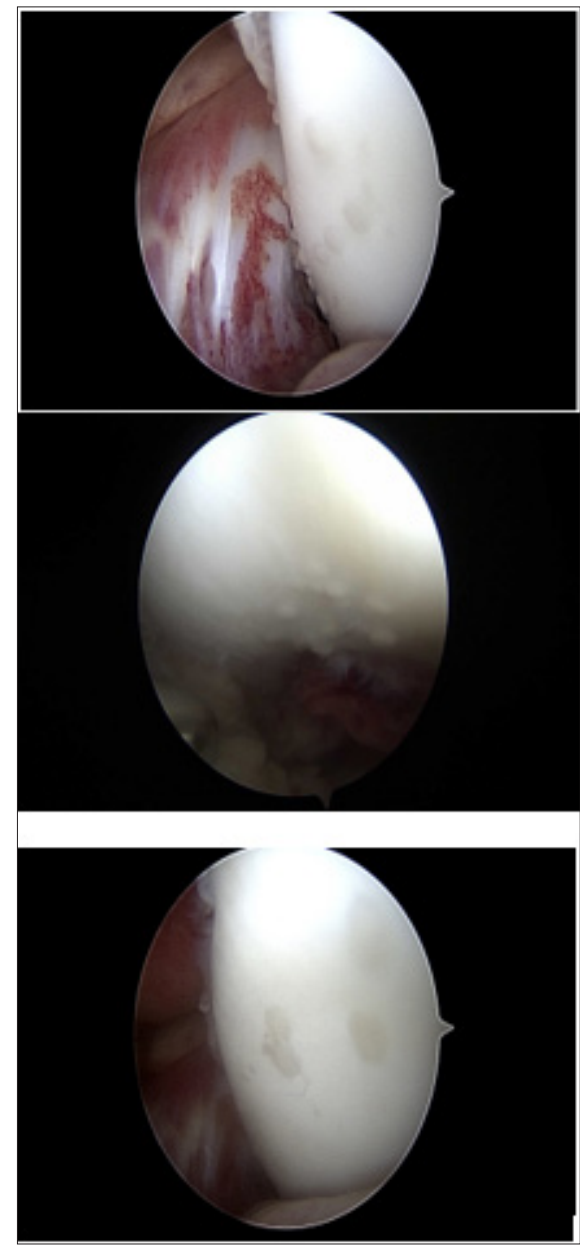

Figure 2: TArthroscopic views of the knee showing plaque formation on the femoral condyles.
Patient was started on IV Micafungin and was taken to the operating room where a formal irrigation and debridement was performed. A large amount of dark, viscous and serosanguinous fluid was encountered and evacuated from the joint along with a large of necrotic looking synovial tissue in the lateral gutter of the knee joint. Following initial I\&D, patient continued to have febrile episodes and rapidly progressive skin rash. Initial medical work up including echocardiogram and CT scans of the abdomen and pelvis showed no evidence of vegetations or hepato-splenic candidiasis. Over the next 2 weeks, our patient subsequently underwent 3 additional open knee irrigations with sharp debridement of the synovium due to failure of response, despite adjustments in antifungal medications, including addition of Amphotericin B. Patient subsequently underwent a complete an arthroscopic synovectomy to better reduce her fungal load. An abundance of white plaques was noted on her femoral condyles that were determined to be yeast colonies on culture, (Figure 2).

In the two weeks following her final surgery, patient continue to have significant pain and swelling to the left knee, however, she remained afebrile. Her overall clinical picture soon began to decline, and repeat echocardiogram demonstrated a pericardial effusion with loculations that was not present on initial examination. Chest radiographs taken 2 weeks apart, (Figure 3), show enlarging of the cardiac silhouette. Her renal function subsequently began to decline as well, secondary to the Amphotericin B. Amphotericin B was discontinued and she was started on Voriconazole. Her oncology and pediatric medical teams decided to initiate a granulocyte transfusion to due to her continued pancytopenia and poor response to medical and surgical treatment of her infection. Her granulocyte transfusion was not well tolerated, and the patient developed respiratory distress in addition to worsening kidney 
function. CBC following her granulocyte transfusion showed peripheral blasts cells that were confirmed by flow cytometry to be relapsed AML. After a family meeting with the medical teams, a decision was made to place the patient on hospice and comfort measures. She was discharged from the hospital and expired at home the following day.
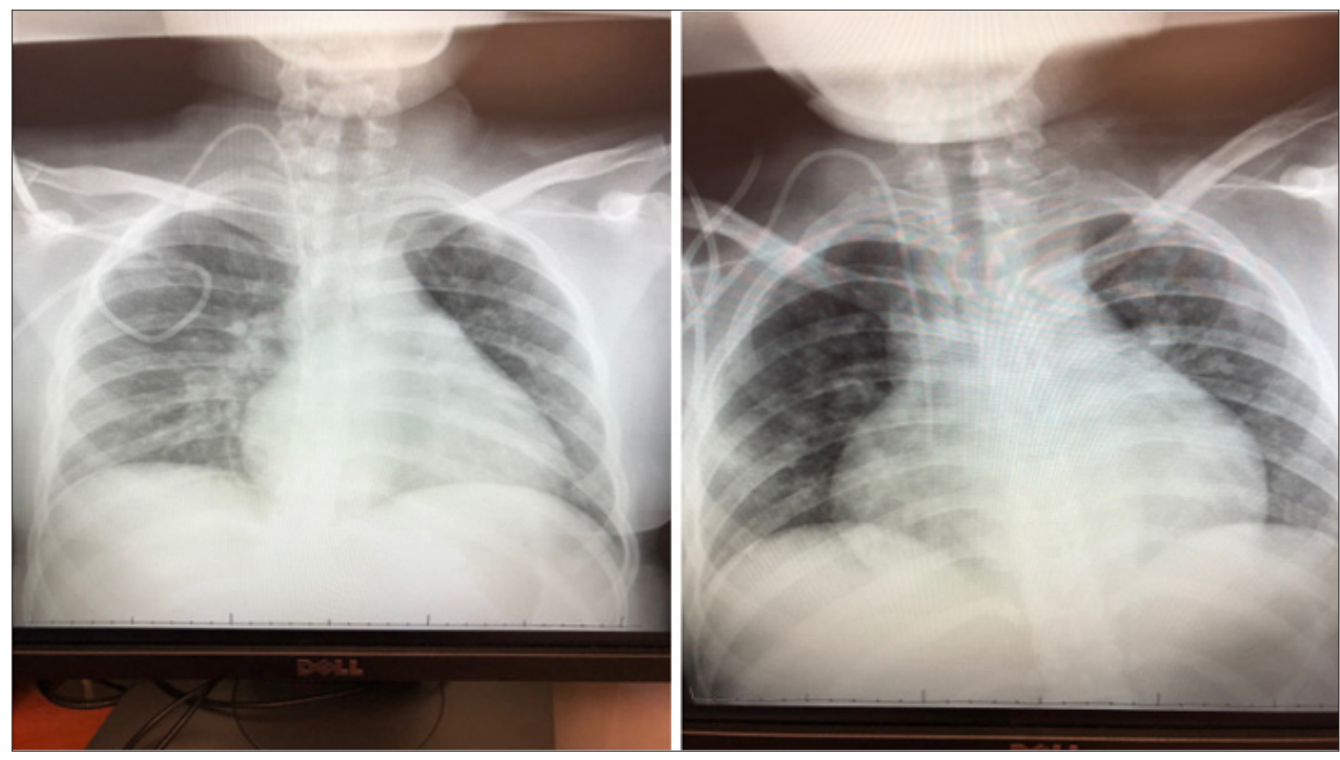

Figure 3: Chest radiographs obtained 2 weeks apart demonstrating enlarged cardiac silhouette.

\section{Discussion}

Septic arthrosis with Candida tropicalis infection is a rare infection in the United States. Patients at risk for developing this infection include those with immunocompromising conditions including malignancy, HIV/AIDS, neonates, patients with prolonged ICU stays with or without mechanical ventilation, those with indwelling central venous catheters, prolonged broad-spectrum antibiotic use, direct inoculation or previous surgery [1-2]. Systemic infections with tropicalis are common in Asian and South American countries, where various studies have shown to be the third most commonly isolated species in patients with systemic candidemia. C. tropicalis produces more persistent infections than $C$. albicans leading to longer hospital stays [3-5,9]. Recent studies have also shown that $C$. tropicalis is becoming increasingly resistant to antifungals as a result of $C$. tropicalis' various virulent factors, including biofilm formation, adhesion molecules, cell wall hydrophobicity as well as extracellular proteases and phospholipases [6]. It has been shown to have a higher mortality rate when compared to all other non-Candida Albicans candidemia (NCAC) species with mortality rates surpassing C. albicans $[1,8]$.

Septic joint arthrosis and osteomyelitis caused by Candida species are typically seeded by hematogenous dissemination, direct inoculation or direct extension from nearby focus of infection. Knee joints are the most common site of infection, owning to the highly vascular synovium, which lacks a limiting basement membrane allowing for easy passage for joint inoculation [10]. In pediatric patients, open epiphyseal plates allow for hematogenously disseminated organisms to extend into the joint as well as the metaphysis, setting the stage for osteomyelitis
$[1,7]$. Candida arthritis presents with a clinical picture similar to bacterial septic arthritis, however, fever may not be a presenting symptom and inflammatory markers may only be moderately elevated, particularly in immunocompromised hosts. The most common presenting symptoms include joint pain and effusion with limitations to range of motion and weightbearing secondary to pain. Surgical findings include thickened fibrotic synovium, cartilage damage and purulence $[9,10]$.

The current literature regarding septic arthritis with $C$. tropicalis in pediatric populations is sparse with the vast majority of case reports emerge from outside of the United States [11-14]. Gamaletsou et al. [1] performed a systematic review of the literature of 112 cases of adult and pediatric with confirmed diagnosis of Candida arthritis. They found that $C$. tropicalis infection occurred in $14 \%$ of the cases identified and was the second most common recovered species behind C. albicans. $36 \%$ of the patients identified in their study pediatric, with the vast majority being neonates. $78 \%$ of the patients were cleared of the infection with either medical therapy alone or in conjunction with surgical intervention. However, while there was no significant difference between patients treated with medical therapy alone or combine with surgery, there were far fewer deaths with the latter. McCullers and Flynn reported on a case of tropicalis arthritis and osteomyelitis in a 5-year-old male following chemotherapy treatment for acute lymphocytic leukemia. This infection was successfully treated with a prolonged course of amphotericin B and rifampin. Their review of the literature found 11 additional cases involving adult and pediatric patients, with 3 originating from their institution. They reported a $11.2 \%$ risk of invasive disease among immunocompromised patients with 
culture positive $C$. tropicalis compared to $2 \%$ risk in those colonized with C. albicans.

The Infectious Disease Society of America set forth clinical practice guidelines for the treatment of Candida septic arthritis. Management of native joint septic candidiasis that received strong recommendation included fluconazole for a minimum of 6 weeks or an echinocandin such as caspofungin, micafungin or anidulafungin for 2 weeks followed by a minimum of 4 weeks of fluconazole. Surgical drainage was indicated for all cases of septic arthritis. The use of intra-articular amphotericin B has been reported, however, this modality remains controversial.

\section{Conclusion}

We present a case of monoarticular $C$. tropicalis arthritis in an adolescent female with relapsed acute myeloid leukemia that was unsuccessfully treated despite aggressive medical and surgical intervention. There are few reports in the literature of septic joint candidiasis caused by $C$. tropicalis in the pediatric population, and to our knowledge, none have been reported in North America involving the adolescent age group that resulted in patient death Most cases reported in the literature have shown $C$. tropicalis septic arthritis and osteomyelitis to be successfully treated with prolonged administration of anti-fungal agents and surgical management. While this condition presents with a clinical picture similar to bacterial arthritis, the clinician should have a high suspicion for Candida arthritis in patients with immunocompromising conditions and with indwelling catheters with new onset joint pain and effusion to reduce patient morbidity and mortality.

\section{References}

1. Gamaletsou MN, Rammaert B, Bueno MA, Sipsas NV, Moriyama B (2015) Candida arthritis: Analysis of 112 pediatric and adult cases. Open Forum of Infectious Disease 3(1): ofv207.

2. Zuza Alves DL, Silva Rocha WP, Chaves GM (2017) An update on Candida tropicalis based on basic and clinical approaches. Front Microbiol 13(8): 1927.
3. Kontoyiannis DP, Vaziri I, Hanna HA, Boktour M, Thornby J (2001) Risk factors for Candida tropicalis fungemia in patients with cancer. Clin Infect Dis 33(10): 1676-1681.

4. Pappas PG, Kauffman CA, David RA, Cornelius JC, Kieren AM (2016) Clinical practice guideline for the management of candidiasis: 2016 update by the infectious disease's society of America. Clinical Infectious Diseases (62)4: e1-e50.

5. Sónia S, Negri M, Henriques M, Oliveira R, Williams DW (2012) Candida glabrata, Candida parapsilosis and Candida tropicalis: Biology, epidemiology, pathogenicity and antifungal resistance. FEMS Microbiology Reviews 36(2): 288-305.

6. JA McCullers, Flynn PM (1998) Candida tropicalis osteomyelitis: Case report and review. Clinical Infectious Diseases 26(4): 1000-1001.

7. Wang HP, Yen YF, Chen WS, Chou YL, Tsai CY (2007) An unusual case of Candida tropicalis and Candida krusei arthritis in a patient with acute myelogenous leukemia before chemotherapy. Clin Rheumatol 26(7): 1195-1197.

8. Fanning S, Mitchell AP (2012) Fungal biofilms. PLoS Pathog 8(4): e1002585.

9. McCarty TP, Pappas PG (2016) Invasive candidiasis. Infect Dis Clin North Am 30(1): 103-124.

10. Krcmery V Jr, Mrazova M, Kunova A, Grey E, Mardiak J (1999) Nosocomial candidaemias due to species other than Candida albicans in cancer patients. Aetiology, risk factors, and outcome of 45 episodes within 10 years in a single cancer institution. Support Care Cancer 7(6): 428-431.

11. Bariteau JT, Warvasz GR, McDonnell M, Fischer SA, Hayda RA (2014) Fungal osteomylietis and septic arthritis. J Am Acad Ortho Surg 22(6): 390-401.

12. Hu XR, He JS, Ye XJ, Zheng WY, Wu WJ (2008) Candida tropicalis arthritis in a patient with acute leukemia. Zhongguo Shi Yan Xue Ye Xue Za Zhi 16(5): 1215-1218.

13. Sim JP, Kho BC, Liu HS, Yung R, Chan JC (2005) Candida tropicalis arthritis of the knee in a patient with acute lymphoblastic leukemia: Successfully treatment with caspofungin. Hong Kong Med J 11(2): 120-123.

14. Vicari P, Feitosa PR, Chauffaille ML, Yamamoto M, Figueiredo MS (2003) Septic arthritis as the first sign of candida tropicalis fungaemia in an acute lymphoid leukemia patient. Braz J Infect Dis 7(6): 426-428.

15. Weisse ME, Person DA, Berkenbaugh JT (1993) Treatment of candida arthritis with flucytosine and amphotericin B. J Perinatol 13(5): 402404 . 